\title{
Clinicopathological Characteristics and Survival Outcomes of Mammary Paget's Disease: A Retrospective Study Based on a Chinese Population
}

\author{
Bo-Yue Han ${ }^{1-3, *}$, Xiao-Li Xu ${ }^{3,4, *}$, Xiu-Zhi Zhu ${ }^{1-3, *}$, Xiang-Chen $\mathrm{Han}^{1-3}$, Xin $\mathrm{Hu}^{2,3}$, Hong Ling $\mathbb{D}^{1,3}$ \\ 'Department of Breast Surgery, Fudan University Shanghai Cancer Center, Fudan University, Shanghai, 200032, People's Republic of China; ${ }^{2}$ Key \\ Laboratory of Breast Cancer in Shanghai, Fudan University Shanghai Cancer Center, Fudan University, Shanghai, 200032, People's Republic of China; \\ ${ }^{3}$ Department of Oncology, Shanghai Medical College, Fudan University, Shanghai, 200032, People's Republic of China; ${ }^{4}$ Department of Pathology, \\ Fudan University Shanghai Cancer Center, Fudan University, Shanghai, 200032, People's Republic of China
}

Correspondence: Xin Hu; Hong Ling, Tel +86 I80I7317652; +86 I80I73 I2656, Email xinhufd@I63.com; linghong98@aliyun.com

*These authors contributed equally to this work

Background: Mammary Paget's disease (PD) is a rare type of breast cancer. Most cases of PD are presented with underlying ductal carcinoma in situ (DCIS) or invasive breast carcinoma (IDC). This study aimed to investigate the clinicopathological characteristics and survival outcomes of PD patients.

Materials and Methods: A total of 406 patients diagnosed with PD with IDC/DCIS at Fudan University Shanghai Cancer Center (FUSCC) were recruited as the PD group, 1218 patients diagnosed with IDC/DCIS alone during the same period were selected as the non-PD group, and the clinicopathological results of these two groups were compared. The Surveillance, Epidemiology, and End Results (SEER) database was used to investigate the clinicopathological features between PD and non-PD patients for validation.

Results: Compared with the non-PD group, the PD group was much more likely to have larger ( $\geq 2 \mathrm{~cm}: 43.1 \% \mathrm{vs} 35.5 \%$, P $<0.001$ ), less hormone receptor (HR)-positive $(68.5 \%$ vs $26.6 \%, \mathrm{P}<0.001)$, more human epidermal growth factor receptor-2 (HER-2)-positive (70.7\% vs $27.5 \%, \mathrm{P}<0.001)$ and higher Ki-67 proportion $(51.5 \%$ vs $42.5 \%, \mathrm{P}<0.001)$ tumors. The HER-2 overexpression subtype accounted for the largest proportion in the PD-IDC group and the lowest proportion in the non-PD-IDC group (54\% vs $8 \%, \mathrm{P}<0.01)$. Moreover, the PD group had significantly worse disease-free survival (DFS) than the non-PD group (5-year DFS: $91.8 \%$ vs $97.3 \%$, P = 0.001 ), and the SEER database showed a similar trend. Univariate and multivariate Cox regression analyses demonstrated that PD was an independent poor-risk factor. Our matched study showed that the PD group had worse survival than the non-PD group after excluding age, HR, HER-2, tumor size and lymph node status.

Conclusion: PD with IDC/DCIS is associated with more aggressive tumor characteristics and worse survival outcomes. More than half of PD breast cancers are HER-2 overexpression subtype. PD is an independent poor-risk factor for breast cancer survival.

Keywords: breast cancer, mammary Paget's disease, clinicopathological characteristic, survival, prognosis, molecular subtype

\section{Background}

Breast cancer is the most common cancer among women worldwide. ${ }^{1}$ Mammary Paget's disease (PD) is a rare type of breast cancer. The incidence of this rare abnormality has been reported to be $0.5-5 \%$ of all breast cancers. ${ }^{2,3} \mathrm{PD}$ is clinically characterized by eczematoid eruption on the nipple and areola, accompanied by other skin changes, such as itching, erythema, bloody nipple discharge, nipple erosion or ulceration, and nipple retraction. ${ }^{4}$ Infiltration of malignant mammary epithelial cells in the nipple epidermis is the main pathological change in PD. ${ }^{5}$

There are two hypothesises proposed to explain the pathogenesis of $\mathrm{PD} .{ }^{4}$ The in situ malignant transformation theory proposes that the Paget's cells are malignant cells that appear in situ independently of any underlying precancerous or 
cancerous condition. ${ }^{6}$ The epidermotropic theory suggests that Paget's cells originate from the underlying ductal tumor and migrate along the lactiferous ducts to reach the nipple epidermis. ${ }^{7}$

Approximately $90 \%$ of mammary PD cases present with underlying ductal carcinoma in situ (DCIS) or invasive breast carcinoma (IDC); only a small proportion of PD cases involve skin changes alone. ${ }^{8}$

Previous studies have reported that the underlying tumor of PD was more prone to negative estrogen receptor (ER), negative progesterone receptor (PR), positive human epidermal growth factor receptor-2 (HER-2) and high histological grade. ${ }^{8-10}$ Additionally, PD with DCIS or IDC may have worse survival than PD alone; ${ }^{11,12}$ furthermore, the presence of PD may be associated with worse outcomes compared with breast cancer alone. ${ }^{13,14}$

Since PD is a rare disease, most studies have included limited numbers. In addition, the underlying mechanisms of PD still remain controversial. Thus, we enrolled 406 women diagnosed as having PD with IDC/DCIS at Fudan University Shanghai Cancer Center (FUSCC) to study whether the presence of PD is associated with clinicopathological characteristics and survival outcomes with underlying IDC/DCIS (PD-IDC/PD-DCIS).

\section{Materials and Methods}

\section{Participant Eligibility}

Four hundred and six patients diagnosed with nipple-areolar PD with underlying IDC/DCIS between January 1, 2004, and December 31, 2015, at FUSCC were recruited as part of the study group (PD group). Controls (non-PD group, $\mathrm{n}=$ 1218) diagnosed with IDC/DCIS without PD during the same period were randomly selected in a 3:1 ratio for patients in the non-PD group. Participants were excluded if any of the following criteria applied: (1) patients diagnosed with stage IV breast cancer or ductal or lobular atypical hyperplasia, sarcomas or phyllodes tumors; (2) patients with a previous or concomitant malignancy; (3) pregnant patients; or (4) patients with situations precluding long-term follow-up.

In addition, we obtained data from the National Cancer Institute's Surveillance, Epidemiology, and End Results (SEER) database collected between January 1, 2010, and December 31, 2015. ${ }^{15}$ We used the histopathology code of the 3rd edition of the International Classification of Oncology (ICD-O-3) to extract 444 patients diagnosed with PD-IDC (code 8541). A total of 253,496 patients with nonspecific types of ductal carcinoma (ICD-O-3 code 8500) were obtained as the control group. Patients with unknown ER PR or HER-2 status were excluded. This retrospective study was approved by the Ethics Committee Review Board of FUSCC (050432).

A matched group was selected by a propensity score matching model according to five variables: age, HR status, HER-2 status, tumor size and lymph node status. After matching, non-PD patients were randomly selected in a strict 3:1 ratio for patients in the PD group.

\section{Data Collection}

To analyze the clinicopathological characteristics of the PD group, the study parameters were as follows: age at diagnosis; pathologic tumor size; lymph node status; histological grade; ER and PR status; expression of HER-2; and treatments (adjuvant chemotherapy, radiotherapy, endocrine therapy and target therapy). An ER- and/or PR-positive status was considered hormone receptor (HR)-positive.

Disease-free survival (DFS) was defined as the time between the first date of diagnosis and any locoregional recurrence, including ipsilateral breast, local/regional lymph nodes of the disease, any contralateral breast cancer, any distant metastasis of the disease, or any secondary malignancy, whichever occurred first. ${ }^{16,17}$ Overall survival (OS) was defined as the time from the first date of diagnosis to death as a result of any cause, and breast cancer-specific survival (BCSS) was defined as the time from the first date of diagnosis to death caused by breast cancer. ${ }^{16,17}$ The time and site of the first detected relapse, and the time and cause of death were recorded via follow-up phone.

Molecular subtypes were based on St. Gallen 2017 Guidelines. ${ }^{18}$ Luminal A: HR+, HER-2 (-), Ki-67<14\%; Luminal B: HR+, HER-2(-), Ki-67 $\geq 14 \%$ or HR+, HER-2(+); HER-2 Overexpression: HR-, HER-2 (+); TNBC (Triple-negative breast cancer): ER-, PR-, HER-2(-). 


\section{Statistical Methods}

$\mathrm{T}$ tests were used to compare age between the PD group and the non-PD group. Pearson chi-square tests were used to compare other clinicopathological variables. OS, BCSS and DFS were compared between the two groups using the Kaplan-Meier method. Univariate and multivariate Cox regression analyses were performed to identify prognostic factors. ${ }^{19}$ A propensity score matching model was used to conduct the matched study. ${ }^{19}$ All tests were two-sided, and a P-value of less than 0.05 was considered statistically significant. SPSS statistical software version 25.0 package (IBM Corporation, Armonk, NY, USA) and R software version 3.5.3. (The R Project for Statistical Computing, https://www. r-project.org/) were used for the calculations and analyses. The R packages "MatchIt", "survminer", "readr", and "survival" with the appropriate libraries were used.

\section{Results}

\section{Clinicopathological Characteristics}

In total, 406 patients were diagnosed with mammary PD during the study period at FUSCC. The mean age at diagnosis was 52.3 years, with a range from 27 to 92 years. Of these recruited PD patients, $82 \%(n=333)$ had concomitant IDC, and $18 \%(\mathrm{n}=73)$ had DCIS. Table 1 shows the clinicopathological characteristics between the PD group and the non-PD group. Patients in the PD group were older than non-PD patients ( 52.3 years vs 50.5 years, $\mathrm{P}=0.004)$. Compared with the non-PD group, the PD group tended to have larger tumors ( $\geq 2 \mathrm{~cm}: 43.1 \%$ vs $35.5 \%, \mathrm{P}<0.001$ ) and more lymph node metastases $(39.6 \%$ vs $37.1 \%, \mathrm{P}=0.043)$. In addition, the $\mathrm{PD}$ group was much more likely to have HR-negative $(68.5 \%$ vs $26.6 \%, \mathrm{P}<0.001)$, HER-2-positive $(70.7 \%$ vs $27.5 \%, \mathrm{P}<0.001)$ and high proportions of Ki-67 (51.5\% vs $42.5 \%, \mathrm{P}<$ 0.001 ) tumors than the control patients in the non-PD group. The SEER database also demonstrated that the PD group tended to have larger tumors ( $\geq 2 \mathrm{~cm}: 49.8 \%$ vs $41.6 \%$, P $<0.001$ ), more lymph node metastases $(49.8 \%$ vs $32.9 \%, \mathrm{P}<$ $0.001)$, and a greater proportion of HR-negative (37.8\% vs $17.0 \%, \mathrm{P}<0.001)$ and HER-2-positive $(59.2 \%$ vs $15.3 \%, \mathrm{P}<$ $0.001)$ tumors than the non-PD group (Table S1).

\section{Molecular Subtypes}

We compared the molecular subtypes between the 333 patients diagnosed as having PD with IDC (PD-IDC group) and 1114 patients diagnosed with IDC alone (non-PD-IDC group). Figure 1 demonstrates that HER-2 overexpression accounted for the largest proportion in the PD-IDC group, while in the non-PD-IDC group, the proportion of HER-2 overexpression was the lowest $(54 \%$ vs $8 \%, \mathrm{P}<0.001)$. The proportion of both TNBC and luminal breast cancer was much smaller in the PD-IDC group (TNBC: $4 \%$ in the PD-IDC group vs $17 \%$ in the non-PD-IDC group, $\mathrm{P}<0.001$; luminal A: $5 \%$ in the PD-IDC group vs $28 \%$ in the non-PD-IDC group, $\mathrm{P}<0.001$; luminal B: $22 \%$ in the PD-IDC group vs $39 \%$ in the non-PD-IDC group, $\mathrm{P}<0.001$ ). A similar trend was observed in the SEER database. Figure S1 shows that HR-negative and HER-2-positive breast cancer, which corresponds to the HER-2 overexpression subtype, accounted for more breast cancer cases in the PD group than in the non-PD group $(29 \%$ vs $5 \%, \mathrm{P}<0.001)$, while HR-negative and HER-2-negative breast cancer (TNBC, $6 \%$ vs $11 \%, \mathrm{P}<0.001$ ) accounted for fewer breast cancer cases.

\section{Survival Analysis}

For all recruited patients at FUSCC, the median follow-up time was 72.6 months (range, 5-189 months). As shown in Figure 2A, the PD group had a significantly worse DFS than the non-PD group ( $\mathrm{P}=0.001,5$-year DFS: $91.8 \%$ vs $97.3 \%$ ). The survival analysis of the SEER database showed a similar result (Figure 2B): the PD group had significantly worse BCSS than the non-PD group ( $\mathrm{P}<0.001$, 5-year DFS: $85.1 \%$ vs $91.3 \%$ ). However, when we compared OS between the PD-FUCSS and PD-SEER groups, we found that the PD-FUSCC group had better survival (Figure S2, $\mathrm{P}<0.001$ ). Next, a subgroup analysis was performed. The PD-IDC group had worse DFS than the PD-DCIS group (Figure S3A, P $=0.039$ ). For different molecular subtypes in the PD-IDC group, the DFS of TNBC patients was the worst, while patients with HER2-overexpression breast cancer survived the best (Figure 3A, P $=0.045$ ). Interestingly, the good prognosis of HER2-overexpression breast cancer was only observed in patients who had used targeted therapy (Figure 3B and C). 
Table I Clinical Characteristics of PD Group and Non-PD Group of FUSCC

\begin{tabular}{|c|c|c|c|}
\hline \multirow[t]{3}{*}{ Patient Characteristics and Treatments } & PD Group & Non-PD Group & \multirow[t]{3}{*}{$\mathbf{P}^{\mathbf{b}}$} \\
\hline & $n=406$ & $n=|2| 8$ & \\
\hline & No. (\%) & No. & \\
\hline Age (mean) & 52.3 & 50.5 & $0.004^{c}$ \\
\hline Tumor size(cm) & & & $<0.001$ \\
\hline$<2$ & $223(54.9 \%)$ & $687(56.4 \%)$ & \\
\hline$\geq 2$ & $175(43.1 \%)$ & $432(35.5 \%)$ & \\
\hline Unknown & $8(2.0 \%)$ & $99(8.1 \%)$ & \\
\hline \multicolumn{4}{|l|}{ Lymph node metastasis (for IDC) } \\
\hline Yes & $132(39.6 \%)$ & $413(37.1 \%)$ & 0.043 \\
\hline No & $197(59.2 \%)$ & $693(62.2 \%)$ & \\
\hline Unknown & $4(1.2 \%)$ & $8(0.7 \%)$ & \\
\hline$H^{a}$ & & & $<0.001$ \\
\hline Positive & $128(31.5 \%)$ & 894 (73.4\%) & \\
\hline Negative & $278(68.5 \%)$ & $324(26.6 \%)$ & \\
\hline HER-2 & & & $<0.001$ \\
\hline Positive & $287(70.7 \%)$ & 335 (27.5\%) & \\
\hline Negative & $57(14.0 \%)$ & 779 (64.0\%) & \\
\hline Unknown & $62(15.3 \%)$ & $104(8.5 \%)$ & \\
\hline Ki-67 & & & $<0.001$ \\
\hline Low $(<14 \%)$ & $108(26.6 \%)$ & $620(50.9 \%)$ & \\
\hline High ( $\geq 14 \%$ ) & 209 (51.5\%) & $518(42.5 \%)$ & \\
\hline Unknown & $89(21.9 \%)$ & $78(6.4 \%)$ & \\
\hline Grade & & & $<0.001$ \\
\hline I & $3(0.7 \%)$ & $49(4.0 \%)$ & \\
\hline 2 & $80(19.7 \%)$ & 649 (53.3\%) & \\
\hline 3 & $287(70.7 \%)$ & $382(31.4 \%)$ & \\
\hline Unknown & 36 (8.9\%) & 138 (| I.3\%) & \\
\hline Tumor Type & & & $<0.001$ \\
\hline IDC & $333(82.0 \%)$ & 1114 (91.5\%) & \\
\hline DCIS & $73(18.0 \%)$ & $104(8.5 \%)$ & \\
\hline Adjuvant Chemotherapy & & & $<0.001$ \\
\hline Yes & $228(56.2 \%)$ & $838(68.8 \%)$ & \\
\hline No & III (27.3\%) & $318(26.1 \%)$ & \\
\hline Unknown & $67(16.5 \%)$ & $62(5.1 \%)$ & \\
\hline Radiotherapy & & & $<0.001$ \\
\hline Yes & $68(16.7 \%)$ & $400(32.8 \%)$ & \\
\hline No & $269(66.3 \%)$ & $740(60.8 \%)$ & \\
\hline Unknown & $69(17.0 \%)$ & $78(6.4 \%)$ & \\
\hline Endocrine therapy & & & $<0.001$ \\
\hline Yes & $92(22.7 \%)$ & 774 (63.5\%) & \\
\hline No & $265(65.3 \%)$ & 370 (30.4\%) & \\
\hline Unknown & 49 (12.1\%) & $74(6.1 \%)$ & \\
\hline Target therapy & & & $<0.001$ \\
\hline Yes & 79 (19.5\%) & 152 (I2.5\%) & \\
\hline No & 195 (48.0\%) & 999 (82.0\%) & \\
\hline Unknown & $132(32.5 \%)$ & 67 (5.5\%) & \\
\hline
\end{tabular}

Notes: ${ }^{a} \mathrm{HR}$ positive: ER (Estrogen Receptor) positive or/and PR (Progesterone Receptor) positive; 'Pearson Chi-square tests between PD Group and Non-PD Group; ${ }^{c}$-tests were used to compare age between PD Group and Non-PD Group.

Abbreviations: PD, Paget's disease; HR, hormone receptor; HER-2, human epidermal growth factor receptor-2; IDC, invasive ductal carcinoma; DCIS, dutal carcinoma in situ. 


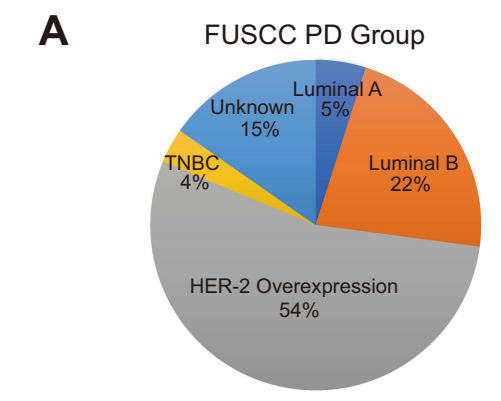

- Luminal $A=$ Luminal $B=$ HER-2 Overexpression
- TNBC $=$ Unknown

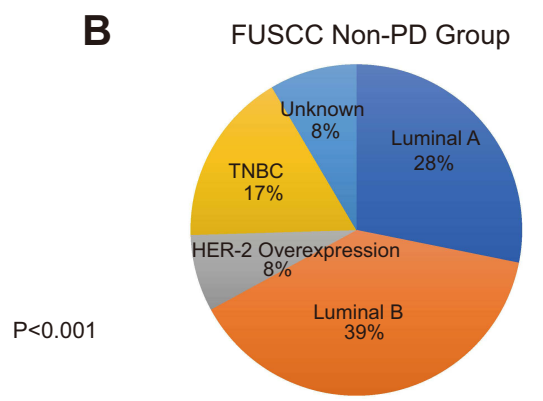

- Luminal A = Luminal B = HER-2 Overexpression

$=$ TNBC $=$ Unknown
C

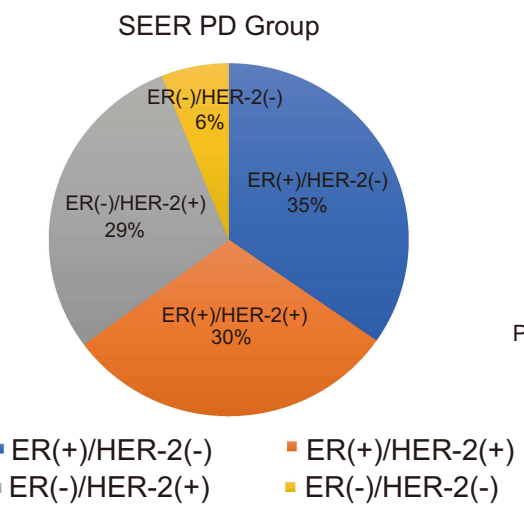

D

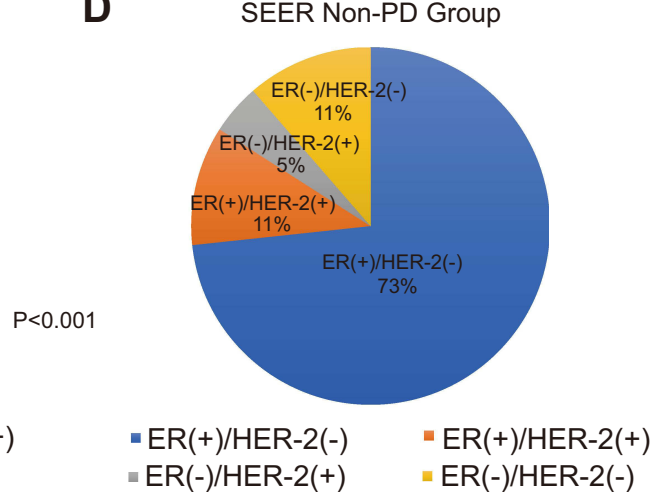

Figure I Molecular subtypes of the PD Group and Non-PD Group of FUSCC, SEER database. (A) Molecular subtypes of the PD group of FUSCC, $n=333$. (B) Molecular subtypes of the Non-PD Group of FUSCC, $n=1$ I 14. (C) Molecular subtypes of the PD Group of SEER database, $n=444$. (D) Molecular subtypes of the Non-PD Group of SEER database, $n=253,496$. The $P$ value was less than 0.00 I, by using Pearson Chi-square tests to compare the distribution of molecular subtypes in PD group (A) and NonPD Group (B) in FUSCC, demonstrating a difference. The $\mathrm{P}$ value was also less than $0.00 \mathrm{I}$, by using Pearson Chi-square tests to compare the distribution of molecular subtypes in PD group (C) and Non-PD Group (D) in SEER database, demonstrating a difference. HR (Hormone Receptor) +: Either ER or PR+. Luminal A: HR+, HER-2 (-), Ki-67<14\%; Luminal B: HR+, HER-2(-), Ki-67 I 4\% or HR+, HER-2(+); HER-2 Overexpression: HR-,HER-2 (+); TNBC (Triple negative breast cancer): ER (-),PR (-),HER-2 (-). Because the SEER database lacks information on Ki-67, molecular subtypes were replaced by HR(+)HER-2(-), HR(+)HER-2(+), HR(-)HER-2(+), HR(-)HER-2(-).

Abbreviations: PD, Paget's disease; ER, estrogen receptor; PR, progesterone receptor; HER-2, human epidermal growth factor receptor-2.

\section{Prognostic Significance}

Univariate and multivariate Cox regression analyses were performed to test the predictive function of clinicopathological factors on DFS (Table 2). Univariate Cox regression analyses demonstrated that age, HR, tumor size, lymph node status and PD were related to poor outcomes in BC patients. After adjusting for confounding variables, PD was confirmed to be an independent factor for DFS (adjusted HR, 1.647; 95\% CI, 1.030-2.639; P = 0.037). Other independent risk factors were age (adjusted HR, 1.595; 95\% CI, 1.041-2.445; P =0.032), HR (adjusted HR, 0.518; 95\% CI, 0.354-0.756; P = 0.001), tumor size (adjusted HR, 1.275; 95\% CI, 1.059-1.535; P = 0.010) and lymph node status (adjusted HR, 3.261; 95\% CI, 2.226-4.776; P < 0.001). To further confirm that PD is an independent poor prognostic factor for breast cancer, we excluded variables such as age, HR, HER-2, tumor size and lymph node status by matching (Table S3). Our matched study revealed that the PD group showed worse survival than the non-PD group $(\mathrm{P}=0.022$, Figure 4).

Furthermore, we explored the prognostic factors of mammary Paget's disease (Table 3). In univariate Cox regression analysis, positive HER-2 status and negative lymph node status were associated with longer DFS. Multivariable Cox regression analysis confirmed that HER-2 status (adjusted HR, 0.338; 95\% CI, 0.185-0.618; P = 0.000 ) and lymph node status (adjusted HR, 2.419; 95\% CI, 1.340-4.368; P =0.003) were independent prognostic factors for PD group. 
A

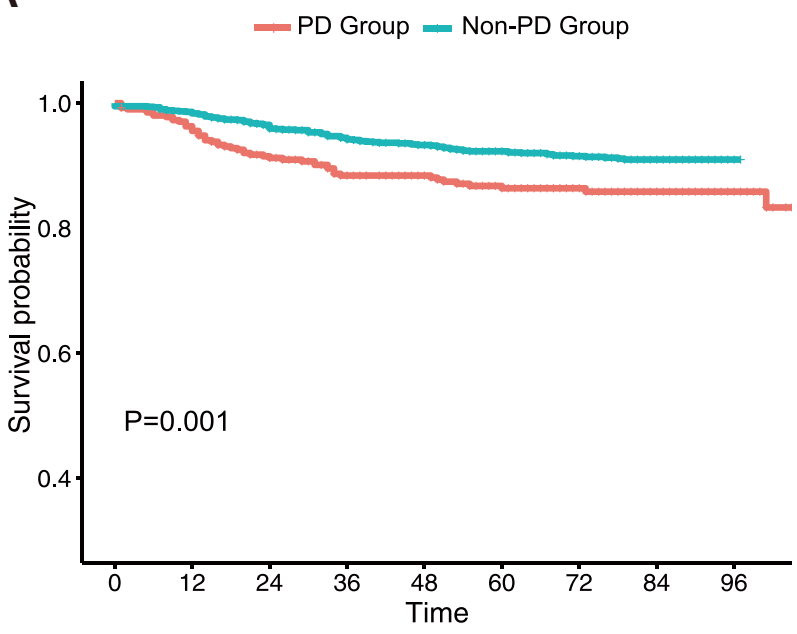

Number at risk
$-\left\{\begin{array}{ccccccccc}406 & 390 & 344 & 307 & 280 & 232 & 163 & 98 & 46 \\ 1218 & 1202 & 1137 & 1076 & 1032 & 992 & 826 & 480 & 27 \\ \hline 0 & 12 & 24 & 36 & 48 & 60 & 72 & 84 & 96 \\ & & \multicolumn{7}{c}{\text { Time }}\end{array}\right.$
B
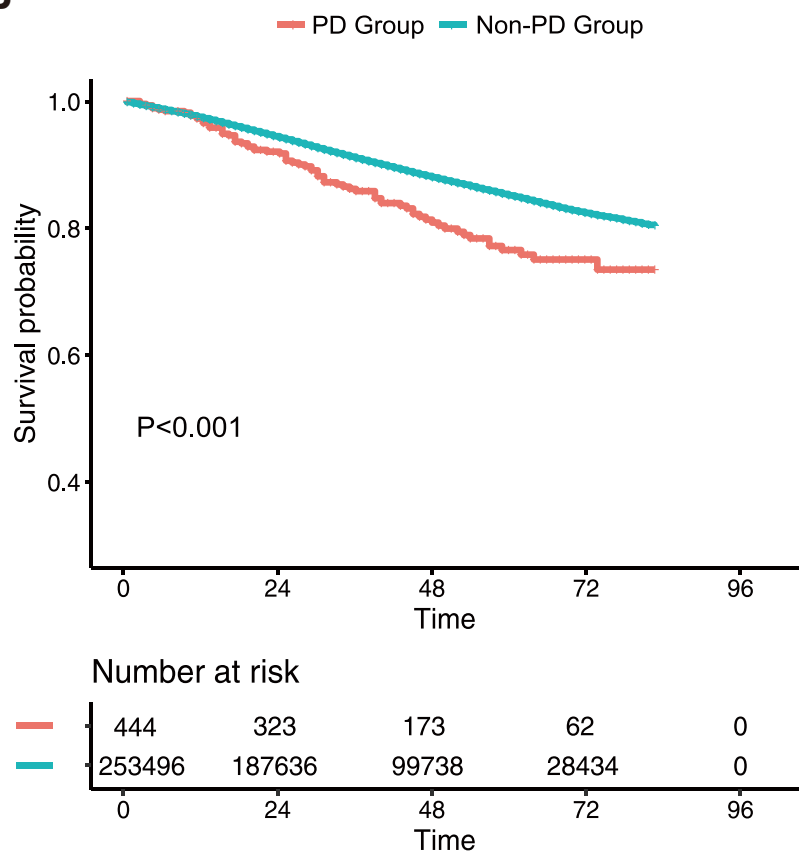

Figure 2 Kaplan-Meier Survival Curves of patients in PD Group and Non-PD Group of FUSCC, SEER database. (A) Comparison of DFS of patients in PD Group and NonPD Group of FUSCC. (B) Comparison of BCSS of patients in PD Group and Non-PD Group of SEER database. The DFS was estimated between PD Group and Non-PD Group of FUSCC by Log rank test with a P value of 0.00 I. The BCSS was estimated between PD Group and Non-PD Group of SEER database by Log rank test with a P value less than 0.001 .

Abbreviations: PD, Paget's disease; DFS, disease free survival; BCSS, breast cancer specific survival.

\section{Discussion}

Since mammary PD was discovered in the 19th century, the mechanism of its pathogenesis has been controversial. To date, there are two mainstream hypotheses of PD origins: epidermotropic and transformation theories. ${ }^{20,21}$ Recently, whole-exome sequencing was performed on paired mammary PD and BC samples. ${ }^{22}$ Distinct gene mutations were detected and explained by independent oncogenic events between the two groups, which might complicate and obscure the association between mammary PD and BC. Meanwhile, for BC patients with PD, there are no specific category data addressing definite management based on version 3.2018 of the current National Comprehensive Cancer Network (NCCN) guidelines (NCCN Guidelines Insights: Breast Cancer, Version 3.2018). Today, PD is not specifically considered, and its treatment is based on the biological characteristics and stage of any underlying cancer and the experience of physicians. Exploring the biological characteristics and clinical prognosis of $\mathrm{BC}$ with or without concomitant PD accurately and objectively is the focus of our study.

Based on our study cohort and SEER database, the PD group was older, and its patients tended to have larger tumors and more lymph node metastases than the non-PD group patients did (Tables 1 and $\underline{\mathrm{S} 1}$ ). This is consistent with Sisti et al's reports that Paget's disease of the breast usually affects older patients. ${ }^{23}$ Besides, the results suggested that patients diagnosed with mammary PD with underlying invasive cancer would have poor tumor characteristics, which is consistent with a study reported by Wong et al. ${ }^{13}$ Part of the reason for this phenomenon could be explained by the relatively rapid progression of PD disease. Lim et al reported that PD was initially mistaken for skin inflammation and ignored by patients, and most patients came to the clinic with relatively advanced disease. ${ }^{24}$ Since $82.0 \%$ of PD patients in our cohort had concomitant IDC, PD needs to attract the attention of doctors and patients, and early detection and consultation are very important. ${ }^{24}$ In addition, BC patients in the PD group were much more likely to be HR negative and HER-2 positive and to have a higher proportion of Ki-67 tumors than BC patients in the non-PD group. Of the four molecular subtypes of BC, HER-2 overexpression accounted for $54 \%$ of cases in the PD-IDC group and $8 \%$ of cases in 
A
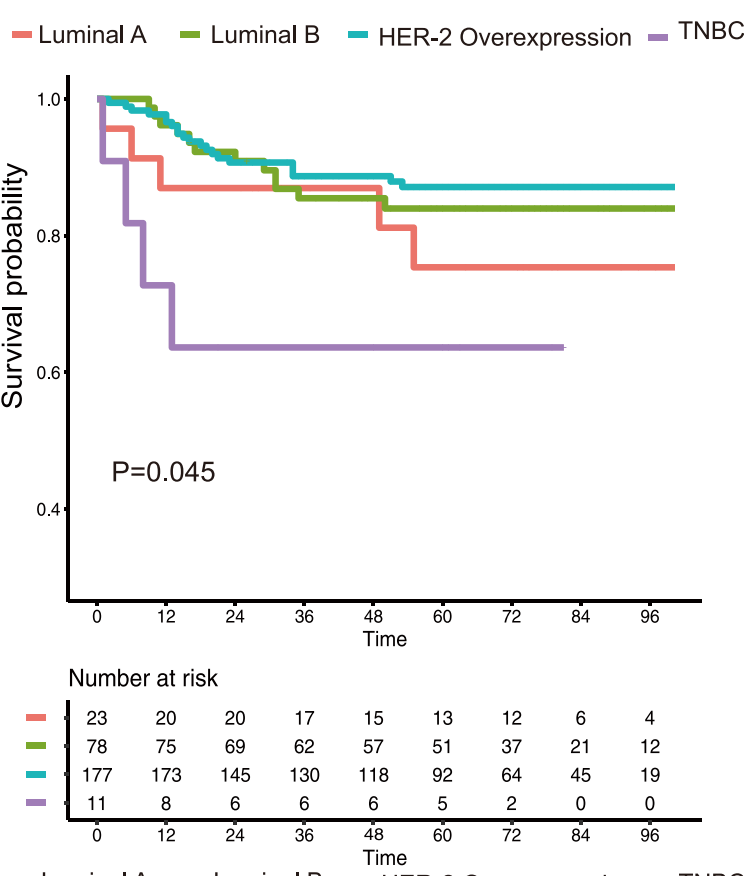

C

- Luminal A $=$ Luminal B $=$ HER-2 Overexpression $=$ TNBC
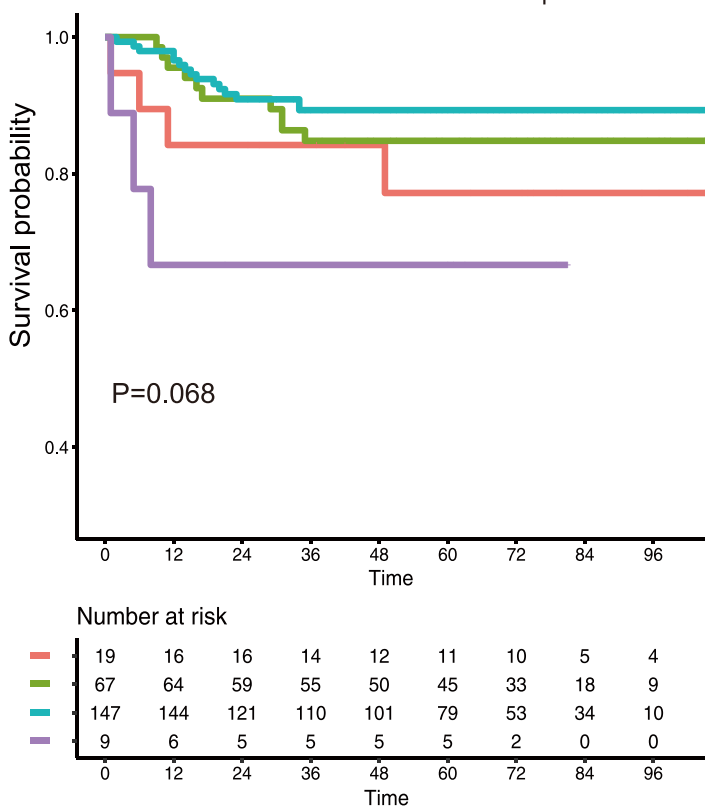

B

- Luminal A - Luminal B - HER-2 Overexpression - TNBC
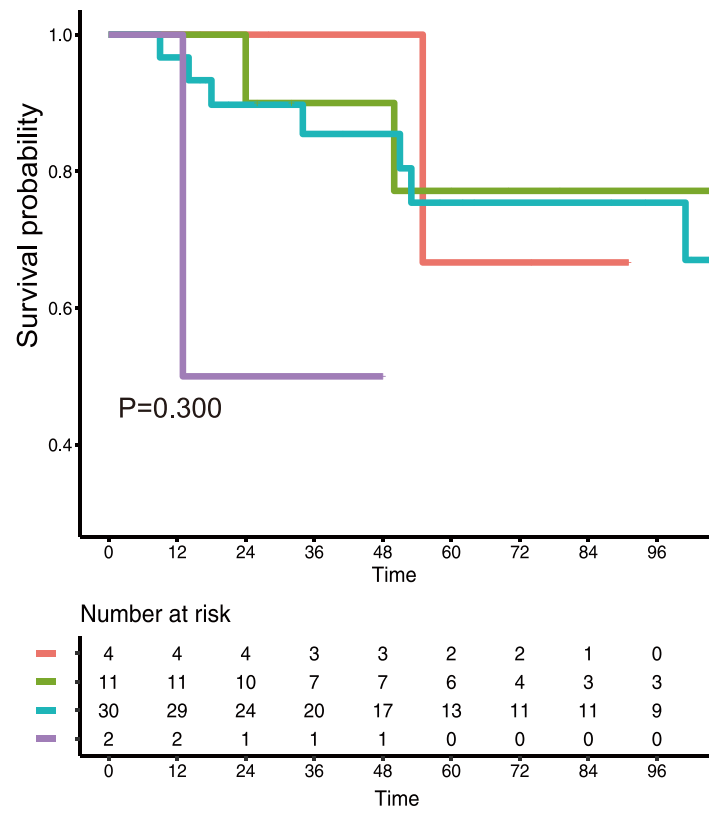

Figure 3 Kaplan-Meier Survival Curves of PD patients in different subgroups of FUSCC. (A) Comparison of DFS according four molecular subtypes: Luminal A, Luminal B, HER-2 Overexpression and TNBC. (B) Disease Free Survival curves of different subgroups before 2010 without anti-HER-2 therapy. (C) Disease Free Survival curves of different subgroups after 2010 with anti-HER-2 therapy. The DFS of $(\mathbf{A})$ was estimated by Log rank test with a P value of 0.045 . The DFS of (B) was estimated by Log rank test with a $P$ value of 0.300 . The DFS of (C) was estimated by Log rank test with a $P$ value of 0.068 .

Abbreviations: PD, Paget's disease; DFS, disease free survival.

the non-PD-IDC group $(\mathrm{P}<0.001)$. Since the different molecular subtypes of $\mathrm{BC}$ have different risk factors, ${ }^{25}$ there are also specific risk factors for the development of PD-IDC; however, currently, those factors remain unknown. ${ }^{26}$

Worse DFS was observed in the PD group than in the non-PD group, as shown by our cohort and SEER database survival analysis (Figure 2A and B). Univariate and multivariate Cox regression analyses showed that PD was an independent factor influencing DFS (Table 2). Our matched group study also confirmed that PD was an independent poor-risk factor. Several previous series have demonstrated that PD negatively influences BC survival, ${ }^{13,27,28}$ but few of these previous studies have been based on Asian populations, or they had small sample sizes. Zhou et.al ${ }^{4}$ performed 
Table 2 Univariate and Multivariate Cox Regression Analyses for DFS Time Among PD and Non-PD Group of FUSCC

\begin{tabular}{|c|c|c|c|c|c|c|}
\hline \multirow[t]{2}{*}{ Variable } & \multirow{2}{*}{$\begin{array}{l}\text { Univariate } \\
\text { Hazard Ratio }\end{array}$} & \multirow[t]{2}{*}{$95 \% \mathrm{Cl}$} & \multirow[t]{2}{*}{$P$ value } & \multirow{2}{*}{$\begin{array}{l}\text { Multivariate } \\
\text { Hazard Ratio }\end{array}$} & \multirow[t]{2}{*}{$95 \% \mathrm{Cl}$} & \multirow[t]{2}{*}{$P$ value } \\
\hline & & & & & & \\
\hline Age(year) & & & 0.017 & & & 0.032 \\
\hline$<40$ & 1 & & & I & & \\
\hline$\geq 40$ & 1.683 & $1.098-2.578$ & & 1.595 & $1.04 I-2.445$ & \\
\hline$H_{R^{a}}$ & & & 0.001 & & & 0.001 \\
\hline Negative & 1 & & & I & & \\
\hline Positive & 0.539 & $0.379-0.767$ & & 0.518 & $0.354-0.756$ & \\
\hline Her-2 & & & 0.987 & & & \\
\hline Negative & 1 & & & & & \\
\hline Positive & 1.003 & $0.702-1.434$ & & & & \\
\hline Tumor size(cm) & & & 0.000 & & & 0.010 \\
\hline$<2$ & 1 & & & I & & \\
\hline$\geq 2$ & 1.509 & $1.261-1.806$ & & 1.275 & $1.059-1.535$ & \\
\hline Lymph node metastasis & & & 0.000 & & & 0.000 \\
\hline No & 1 & & & I & & \\
\hline Yes & 3.612 & $2.500-5.219$ & & 3.261 & $2.226-4.776$ & \\
\hline With Paget's Disease & & & 0.005 & & & 0.037 \\
\hline No & 1 & & & 1 & & \\
\hline Yes & 1.702 & $1.169-2.479$ & & 1.647 & $1.030-2.639$ & \\
\hline
\end{tabular}

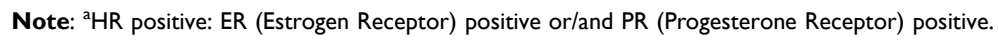

Abbreviations: PD, Paget's disease; HR, hormone receptor; HER-2, human epidermal growth factor receptor-2; Cl, confidence interval.

a matched case-control study between 85 Chinese BC patients with PD and a matched group of 85 BC patients without PD and observed poor OS and DFS in the PD group. Our cohort study was based on a natural Chinese cohort with relatively large samples, which might be more reflective of the clinical characteristics of Asian PD patients. In addition,

- PD Group $\quad$ - Non-PD Group

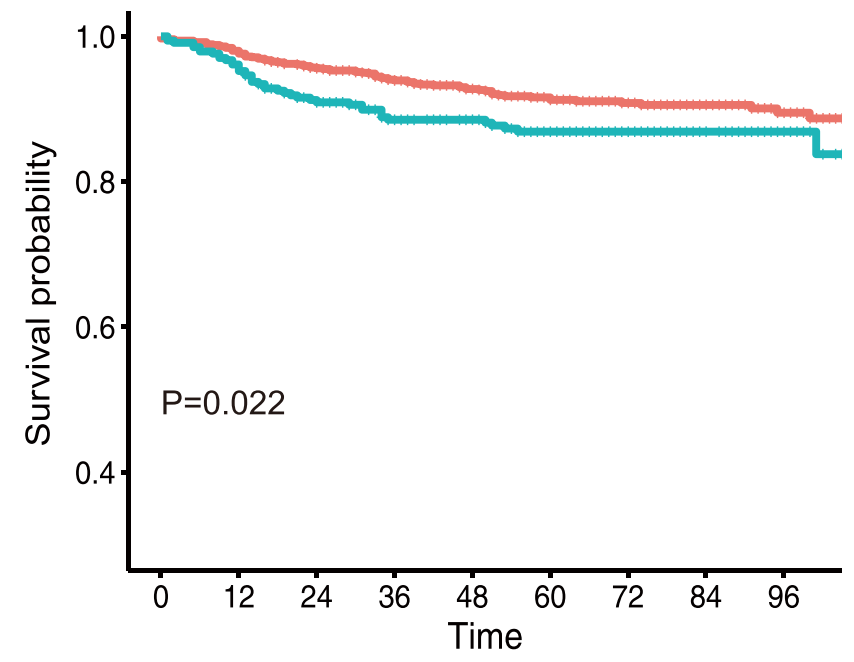

Number at risk

$=$\begin{tabular}{ccccccccc}
1005 & 985 & 935 & 882 & 713 & 534 & 362 & 250 & 143 \\
335 & 322 & 282 & 253 & 230 & 188 & 132 & 81 & 38 \\
\hline 0 & 12 & 24 & 36 & 48 & 60 & 72 & 84 & 96 \\
& & \multicolumn{7}{c}{ Time }
\end{tabular}

Figure 4 Kaplan-Meier Survival Curves between PD Group and Non-PD Group (Matched Group) of FUSCC. The DFS was estimated by Log rank test with a P value of 0.022. Abbreviations: PD, Paget's disease; DFS, disease free survival. 
Table 3 Univariate and Multivariate Cox Regression Analyses for DFS Time Among PD Group of FUSCC

\begin{tabular}{|c|c|c|c|c|c|c|}
\hline \multirow[t]{2}{*}{ Variable } & \multirow{2}{*}{$\begin{array}{l}\text { Univariate } \\
\text { Hazard Ratio }\end{array}$} & \multirow[t]{2}{*}{$95 \% \mathrm{Cl}$} & \multirow[t]{2}{*}{$P$ value } & \multirow{2}{*}{$\begin{array}{l}\text { Multivariate } \\
\text { Hazard Ratio }\end{array}$} & \multirow[t]{2}{*}{$95 \% \mathrm{Cl}$} & \multirow[t]{2}{*}{$P$ value } \\
\hline & & & & & & \\
\hline Age(year) & & & 0.139 & & & \\
\hline$<40$ & I & & & & & \\
\hline$\geq 40$ & 0.600 & $0.305-1.181$ & & & & \\
\hline $\mathbf{H R}^{\mathrm{a}}$ & & & 0.360 & & & \\
\hline Negative & I & & & & & \\
\hline Positive & 1.319 & $0.729-2.385$ & & & & \\
\hline Her-2 & & & 0.000 & & & 0.000 \\
\hline Negative & I & & & I & & \\
\hline Positive & 0.305 & $0.167-0.555$ & & 0.338 & $0.185-0.618$ & \\
\hline Tumor size(cm) & & & 0.291 & & & \\
\hline$<2$ & I & & & & & \\
\hline$\geq 2$ & 1.403 & $0.749-2.630$ & & & & \\
\hline Lymph node metastasis & & & 0.001 & & & 0.003 \\
\hline No & I & & & I & & \\
\hline Yes & 2.663 & $|.48|-4.79 \mid$ & & 2.419 & $1.340-4.368$ & \\
\hline
\end{tabular}

Note: ${ }^{a} H R$ positive: ER (Estrogen Receptor) positive or/and PR (Progesterone Receptor) positive.

Abbreviations: PD, Paget's disease; HR, hormone receptor; HER-2, human epidermal growth factor receptor-2; Cl, confidence interval.

we observed that the OS in our group was better than that in the SEER group, and this phenomenon may be due to the presence of different races with different PD tumor characteristics (Table S2).

It is interesting to note that more than half of the PD breast cancers were HER-2 overexpression subtypes. Moreover, up to $70.7 \%$ of patients in the PD group had HER-2-positive tumors. This is consistent with Wachter el al's reports, which attracted our attention. ${ }^{26}$ The univariate and multivariate Cox regression analyses in PD group considered HER-2 status as a significant prognostic factor. Furthermore, our matched study demonstrated that PD was an independent poorrisk factor after balancing HER-2 status. It seems that the worst survival of the PD group was caused by PD's intrinsic features other than HER-2 status. Additionally, why the HER-2 overexpression subtype accounts for such a high proportion of cases in the PD group requires further research to explain.

To date, few studies have specifically focused on the relationship between molecular subtype and prognosis as well as anti-Her-2 therapy in mammary PD. We explored the survival prognosis of PD for four different molecular subtypes and found that the DFS of TNBC patients was the worst in the PD-IDC group, while patients with HER-2-overexpressing breast cancer survived the best (Figure $3 \mathrm{~A}, \mathrm{P}=0.045$ ). Taking anti-Her-2 therapy into account, we divided our PD-IDC group into two groups based on whether or not they received targeted therapy. Interestingly, a good prognosis of HER-2 overexpression in breast cancer was only observed in patients who had used targeted therapy (Figure S3B and $\underline{\text { ) }}$. This result indicated that HER-2-overexpression BC patients with PD could benefit from targeted therapy against Her-2. The Her-2 state has been tested in the SEER database since 2010; consequently, some of the analyses based on the SEER database that spanned the period around 2010 had apparently been limited in their analysis of Her- $2,{ }^{28}$ which was why their conclusions somewhat differed from ours.

The present study had some limitations. Considering the Her-2 status, our patients were consecutively recruited for this study from 2004 to 2015, while the SEER database was constructed after 2010. Different time points might influence the strength of our results.

\section{Conclusions}

Our study may provide a stronger understanding of $\mathrm{BC}$ with PD. More than half of the PD breast cancers are the HER-2 overexpression subtype. In addition, PD with IDC/DCIS is associated with more aggressive tumor characteristics and worse survival outcomes. These results can inform early detection, therapeutic intervention, and prognosis for patients with PD. However, prospective clinical studies with larger samples are needed to support these findings. 


\section{Data Sharing Statement}

The data used in this study are available free of charge online at www.seer.cancer.gov on request. All data generated or analyzed during this study are included in this published article.

\section{Ethics Approval and Consent to Participate}

This study did not involve animals. All procedures performed in studies involving human participants were in accordance with the ethical standards of the institutional and/or national research committee and with the 1964 Helsinki Declaration and its later amendments or comparable ethical standards. This retrospective study was approved by the Ethics Committee Review Board of Fudan University Shanghai Cancer Center (050432). The need to obtain informed consent was waived, as the study was a retrospective study and there was no additional risk to patients. All data were anonymized to maintain patient privacy.

\section{Author Contributions}

All authors made a significant contribution to the work reported, whether that is in the conception, study design, execution, acquisition of data, analysis and interpretation, or in all these areas; took part in drafting, revising or critically reviewing the article; gave final approval of the version to be published; have agreed on the journal to which the article has been submitted; and agree to be accountable for all aspects of the work.

\section{Funding}

There is no funding to report.

\section{Disclosure}

The authors declare no conflicts of interest.

\section{References}

1. Siegel RL, Miller KD, Jemal A. Cancer statistics, 2020. CA Cancer J Clin. 2020;70(1):7-30. doi:10.3322/caac.21590

2. Fu W, Mittel VK, Young SC. Paget disease of the breast analysis of 41 patients. Am J Clin Oncol. 2001;24(4):397-400. doi:10.1097/00000421200108000-00019

3. Dalberg K, Hellborg H, Warnberg F. Paget's disease of the nipple in a population based cohort. Breast Cancer Res Treat. 2008;111(2):313-319. doi:10.1007/s10549-007-9783-5

4. Zhou H, Lu K, Zheng L, et al. Prognostic significance of mammary Paget's disease in Chinese women: a 10-year, population-based, matched cohort study. Onco Targets Ther. 2018;11:8319-8326. doi:10.2147/OTT.S171710

5. Lohsiriwat V, Martella S, Rietjens M, et al. Paget's disease as a local recurrence after nipple-sparing mastectomy: clinical presentation, treatment, outcome, and risk factor analysis. Ann Surg Oncol. 2012;19(6):1850-1855. doi:10.1245/s10434-012-2226-5

6. Lopes Filho LL, Lopes IM, Lopes LR, Enokihara MM, Michalany AO, Matsunaga N. Mammary and extramammary Paget's disease. An Bras Dermatol. 2015;90(2):225-231. doi:10.1590/abd1806-4841.20153189

7. Sandoval-Leon AC, Drews-Elger K, Gomez-Fernandez CR, Yepes MM, Lippman ME. Paget's disease of the nipple. Breast Cancer Res Treat. 2013;141(1):1-12. doi:10.1007/s10549-013-2661-4

8. Chen CY, Sun LM, Anderson BO. Paget disease of the breast: changing patterns of incidence, clinical presentation, and treatment in the U.S. Cancer. 2006;107(7):1448-1458. doi:10.1002/cncr.22137

9. Wu Q, Ding X, Li J, et al. Surgical treatment in Paget's disease with invasive ductal carcinoma: an observational study based on SEER. Sci Rep. 2017;7:45510. doi:10.1038/srep45510

10. Taffurelli M, Santini D, Al-Balas H, Serra M, Al-Balas M. Clinical and biological characteristics of breast cancer in women with mammary Paget's disease: a retrospective study of 36 cases. Surg Gastroenterol Oncol. 2020;25(5):236.

11. Durkan B, Bresee C, Bose S, Phillips EH, Dang CM. Paget's disease of the nipple with parenchymal ductal carcinoma in situ is associated with worse prognosis than Paget's disease alone. Am Surg. 2013;79(10):1009-1012. doi:10.1177/000313481307901011

12. Ortiz-Pagan S, Cunto-Amesty G, Narayan S, et al. Effect of Paget's disease on survival in breast cancer: an exploratory study. Arch Surg. 2011;146 (11):1267-1270. doi:10.1001/archsurg.2011.278

13. Wong SM, Freedman RA, Sagara Y, et al. The effect of Paget disease on axillary lymph node metastases and survival in invasive ductal carcinoma. Cancer. 2015;121(24):4333-4340. doi:10.1002/cncr.29687

14. Ling H, Hu X, Xu XL, Liu ZB, Shao ZM. Patients with nipple-areola Paget's disease and underlying invasive breast carcinoma have very poor survival: a matched cohort study. PLoS One. 2013;8(4):e61455. doi:10.1371/journal.pone.0061455

15. Yang J, Li Y, Liu Q, et al. Brief introduction of medical database and data mining technology in big data era. J Evid Based Med. 2020;13(1):57-69. doi:10.1111/jebm.12373

16. Hudis CA, Barlow WE, Costantino JP, et al. Proposal for standardized definitions for efficacy end points in adjuvant breast cancer trials: the STEEP system. J Clin Oncol. 2007;25(15):2127-2132. doi:10.1200/JCO.2006.10.3523 
17. Buzdar AU, Suman VJ, Meric-Bernstam F, et al. Disease-free and overall survival among patients with operable HER2-positive breast cancer treated with sequential vs concurrent chemotherapy: the ACOSOG Z1041 (Alliance) randomized clinical trial. JAMA Oncol. 2019;5(1):45-50. doi:10.1001/jamaoncol.2018.3691

18. Curigliano G, Burstein HJ, Winer EP, et al. De-escalating and escalating treatments for early-stage breast cancer: the St. Gallen International Expert Consensus Conference on the primary therapy of early breast cancer 2017. Ann Oncol. 2017;28(8):1700-1712. doi:10.1093/annonc/mdx308

19. Wu WT, Li YJ, Feng AZ, et al. Data mining in clinical big data: the frequently used databases, steps, and methodological models. Mil Med Res. 2021;8(1):44. doi:10.1186/s40779-021-00338-z

20. Yim JH. Underlying pathology in mammary Paget's disease. Ann Surg Oncol. 1997;4(4):287-292. doi:10.1007/BF02303576

21. Lagios MD. Paget's disease of the nipple alternative management in cases without or with minimal extent of underlying breast carcinoma. Cancer. 1984;54(3):545-551. doi:10.1002/1097-0142(19840801)54:3<545::AID-CNCR2820540327>3.0.CO;2-M

22. Zhang G, Zhou S, Zhong W, et al. Whole-exome sequencing reveals frequent mutations in chromatin remodeling genes in mammary and extramammary Paget's diseases. J Invest Dermatol. 2019;139(4):789-795. doi:10.1016/j.jid.2018.08.030

23. Sisti A, Huayllani MT, Restrepo DJ, et al. Paget disease of the breast: a national retrospective analysis of the US population. Breast Dis. 2020;39(34):119-126. doi:10.3233/BD-200439

24. Lim HS, Jeong SJ, Lee JS, et al. Paget disease of the breast: mammographic, US, and MR imaging findings with pathologic correlation. Radiographics. 2011;31(7):1973-1987. doi:10.1148/rg.317115070

25. Holm J, Eriksson L, Ploner A, et al. Assessment of breast cancer risk factors reveals subtype heterogeneity. Cancer Res. 2017;77(13):3708-3717. doi:10.1158/0008-5472.CAN-16-2574

26. Wachter DL, Wachter PW, Fasching PA, et al. Characterization of molecular subtypes of Paget disease of the breast using immunohistochemistry and in situ hybridization. Arch Pathol Lab Med. 2019;143(2):206-211. doi:10.5858/arpa.2017-0578-OA

27. Muttarak M, Siriya B, Kongmebhol P, Chaiwun B, Sukhamwang N. Paget's disease of the breast: clinical, imaging and pathologic findings: a review of 16 patients. Biomed Imaging Interv J. 2011;7(2):e16. doi:10.2349/biij.7.2.e16

28. Chen S, Chen H, Yi Y, et al. Comparative study of breast cancer with or without concomitant Paget disease: an analysis of the SEER database. Cancer Med. 2019;8(8):4043-4054. doi:10.1002/cam4.2242

\section{Publish your work in this journal}

Cancer Management and Research is an international, peer-reviewed open access journal focusing on cancer research and the optimal use of preventative and integrated treatment interventions to achieve improved outcomes, enhanced survival and quality of life for the cancer patient. The manuscript management system is completely online and includes a very quick and fair peer-review system, which is all easy to use. Visit http://www.dovepress.com/testimonials.php to read real quotes from published authors.

Submit your manuscript here: https://www.dovepress.com/cancer-management-and-research-journal 